\title{
Predicting Imaging Outcomes in Acute Stroke Therapy-Comparison of Magnetic Resonance Imaging and Computed Tomography
}

\author{
Deepa Krishnaswamy ${ }^{1}$ Seetharaman Cannane ${ }^{2}$ Meena Nedunchelian ${ }^{3}$ Shriram Varadharajan ${ }^{4}$ \\ Santhosh Poyyamoli ${ }^{4}$ Pankaj Mehta ${ }^{4}$ Mathew Cherian ${ }^{4}$
}

\footnotetext{
${ }^{1}$ Department of Radio-diagnosis, Ahalia Diabetes Hospital, Palakkad, Kerala, India

${ }^{2}$ Department of Radiology, KMCH institute of Health Sciences and Research, Coimbatore, Tamil Nadu, India

${ }^{3}$ Ganga Hospital, Coimbatore, Tamil Nadu, India

${ }^{4}$ Department of Radiodiagnosis, Kovai Medical Center and Hospitals, Coimbatore, Tamil Nadu, India
}

Address for correspondence Dr. Cannane Seetharaman, DMRD, DNB, Assistant Professor, Department of Radiology, $\mathrm{KMCH}$ institute of Health Sciences and Research, Coimbatore, Tamil Nadu, India (e-mail: drcseetharaman@gmail.com).

J Clin Interv Radiol ISVIR 2022;6:83-89.

\begin{abstract}
Keywords

- Stroke

- reperfusion

- CT

- MRI

- predictive value

Background Imaging of acute stroke patients in emergency settings is critical for treatment decisions. Most commonly, CT with CTA is used worldwide for acute stroke. However, MRI may be advantageous in certain settings. With advancements in endovascular clot retrieval techniques, there is a need to identify and use the best possible imaging for the diagnosis and outcome prediction of hyperacute stroke.

Methods This mixed retrospective and prospective observational study was conducted over 2 years in patients who underwent reperfusion therapies. Patients were included in this study if they had a baseline as well as follow-up noncontrast CT and diffusion-weighted imaging (DWI) MRI. We compared them for estimating final infarct size and outcomes after reperfusion therapy.

Results A total of 86 patients were included in the study. Baseline DWI found new infarcts in 33 patients compared to baseline CT. Sensitivity and specificity of CT and DWI in predicting the final infarct size was $75.3 \%$ and $76.9 \%$ and $97.2 \%$ and $92.3 \%$, respectively. A positive correlation of $51.2 \%$ and $84.4 \%$ was noted between b-CT Alberta stroke programme early CT score (ASPECTS) and b-DWI with 72 hours DWI ASPECTS, respectively $(p<0.001)$. The positive predictive value of CT was $94.8 \%$ and DWI was 98.6\%. None of the patients had reversible hyperintensities in the follow-up DWI. Conclusion MRI is more sensitive and specific than noncontrast $C T$ in predicting final infarct volume. It predicts final outcomes better and could be an alternative if available in acute stroke settings.
\end{abstract}

published online October 5, 2021
DOI https://doi.org/ $10.1055 / \mathrm{s}-0041-1736085$. ISSN 2457-0214.
(C) 2021. Indian Society of Vascular and Interventional Radiology. All rights reserved.

This is an open access article published by Thieme under the terms of the Creative Commons Attribution-NonDerivative-NonCommercial-License, permitting copying and reproduction so long as the original work is given appropriate credit. Contents may not be used for commercial purposes, or adapted, remixed, transformed or built upon. (https://creativecommons.org/ licenses/by-nc-nd/4.0/)

Thieme Medical and Scientific Publishers Pvt. Ltd., A-12, 2nd Floor, Sector 2, Noida-201301 UP, India 


\section{Introduction}

Stroke is one of the most common causes of morbidity and mortality all over the world with a prevalence ranging from 44.29 to $559 / 100,000$ persons in different parts of India during the past decade. The incidence rate is 105 to $152 / 100,000$ persons per year, according to recent population-based studies ${ }^{1}$

Rapid imaging-based triage and treatment decisions for interventions are the cornerstone of acute stroke management. When diagnosed and treated in the hyperacute stage, the ischemic changes in the brain parenchyma are potentially reversible, thus giving the patient a high chance of leading a near normal life in the future. Remarkable improvement in outcomes have been demonstrated in the recent years with the use of endovascular clot retrieval devices. However, these procedures are expensive, and the cost versus benefit should be feasible for the vast majority of patients in Indian subcontinent who have to finance their own treatment. Evidently, there is a need to use the best possible imaging modality for the diagnosis and outcome prediction of hyperacute stroke.

Current stroke guidelines ${ }^{2}$ are based on the initial major endovascular trials, which were mainly CT- and computed tomography angiography (CTA) based, because of wider availability and cost-effectiveness of the modality. The Alberta stroke programme early CT score (ASPECTS) ${ }^{3-5}$ and presence of large vessel occlusion (LVO) are recommended as the most important criteria for patient selection for thrombectomy in hyperacute presentations. However, more recent endovascular trials have used perfusion imaging, including MRI-based techniques for patient selection in delayed stroke presentations; further, MRI-based diffusion-weighted imaging-fluid-attenuated inversion recovery (DWI-FLAIR) mismatch was used to decide thrombolysis recently. It has also been shown that MRI-based selection does not impair treatment or patient outcomes. ${ }^{6}$ Being an MRI-based center with $24 \times 7$ availability, we decided to compare the diagnostic performance of MRI versus CT in acute stroke.

We compared the predictive values of CT and MRI in terms of ASPECTS for estimating the final infarct size and patient outcomes after reperfusion therapy. We also looked for the reversibility of the DWI changes in patients presenting within 6 hours of stroke onset.

\section{Materials and Methods}

This study included nonconsecutive patients with clinical or radiological diagnosis of stroke from a mixed retrospective and prospective cohort between the period of June 2016 to June 2018. All such ischemic stroke patients who came to our hospital within 6 hours of clinical presentation, in whom a noncontrast CT and DWI MRI were performed at the time of presentation, who underwent a successful reperfusion therapy, either with mechanical thrombectomy or thrombolysis, and who had a follow-up CT and DWI MRI between 48 to 72 hours following reperfusion were included in the study. Patients who had suboptimal reperfusion

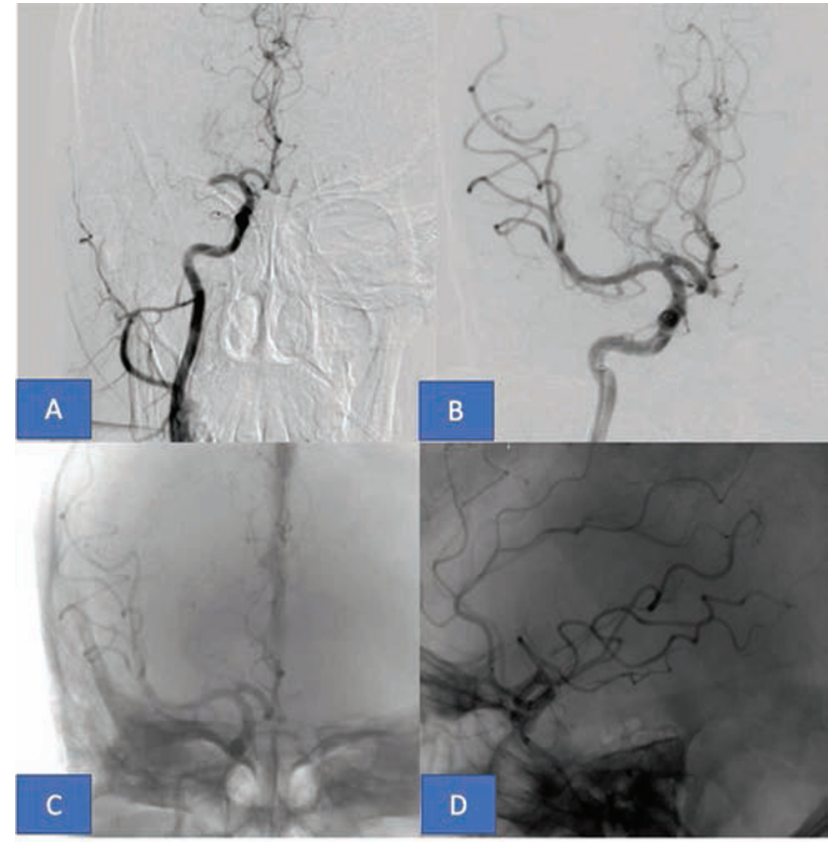

Fig. 1 Angiographic images of patient with right MCA occlusion (A) after mechanical thrombectomy shows successful reperfusion (TICI 2b) with more than $50 \%$ filling of distal territory in both AP and lateral projections (B-D)

and contraindications for performing MRI were excluded from the study.

Successful reperfusion was defined as either modified thrombolysis in cerebral infarction (TICI) $2 \mathrm{~b}$ or more recanalization (partial filling-more than 50\% of the downstream involved territory, as shown in - Fig. 1) on postprocedural angiogram in patients undergoing mechanical thrombectomy, improvement in National Institutes of Health Stroke Scale (NIHSS) clinical score $>4$ points from baseline, or improvement in motor power and speech in those undergoing thrombolysis. ${ }^{7,8}$ Early successful reperfusion has been shown to be associated with better clinical outcomes at 3 months, thus angiographic demonstration of the recanalized vessel post procedure and/or substantial clinical improvement were taken as surrogate markers of the same during acute hospitalization in this study.

CT examinations were performed in a 64 slice multidetector CT (GE Lightspeed VCT). Slice thickness of 0.625 at $120 \mathrm{Kv}$ with a matrix size of $512 \times 512$. CT was performed within 6 hours of onset of symptoms. MRI was performed on both 3 / 1.5 Tesla MRI scanners (Siemens/Philips, respectively). The sequences used were DWI, magnetic resonance angiography (MRA), arterial spin labelling (ASL), susceptibility-weighted imaging (SWI) and FLAIR. Timings for the various sequences (minutes:seconds) are as follows: Siemens 3T MRI-DWI (1:07), MRA Brain (3:17), MRA neck (4:13), SWI (1:49), FLAIR (2:06) and ASL (1:18), while in Philips 1.5 T MRI-DWI (0:56), MRA brain (2:21), MRA neck (4:10), SWI (2:28), FLAIR (2:12) and ASL (3:56)

Most of the emergency stroke code activations in our institute are primarily done in the $1.5 \mathrm{~T}$ Philips MRI, which is next to emergency triage and casualty CT. However, some 
patients also undergo scans in 3T Siemens MRI due to local logistics. Treatment decisions are invariably made after DWI and MRA (within approximately 10 minutes), while rest of the sequences are completed if patient attenders delay consent for treatment or in case of specific patients with delayed presentations such as wake up stroke or unknown time of onset, where additional sequences such as perfusion are needed. Sometimes, hemorrhagic strokes and stroke mimics may also need additional sequences.

\section{Image Analysis and Data Collection}

A blinded review was done by presenting images in a random fashion with regards to both temporal status of the imaging (baseline and follow up) as well as between different patients. This was analyzed by a radiologist of 3 years' experience and the ASPECTS score was recorded for each instance. For the purpose of bringing out the differences in ASPECT score between different modalities, we divided the ASPECT score into three categories ( 3 to 5, 6 to 8, 9 to 10). Reversibility of DWI changes after reperfusion therapy was also analyzed.

Demographic and clinical data including age, sex, time of onset, data pertaining to territory, NIHSS score, and prestroke modified Rankin's scale (mRS) were collected.

\section{Outcome Parameters}

Postrecanalization imaging and clinical analysis included CT and MR ASPECTS done between 48 to 72 hours after therapy which represents true infarct size and mRS at 90 days, respectively.

\section{Statistical Analysis}

The data was entered into the SPSS spreadsheet and verified twice. The analysis was performed in SPSS version 16.0 for Windows. Descriptive analysis such as mean, standard deviation (SD), and percentage were used to exhibit the clinical parameters considered in the research pro-forma. Correlation analysis was used to validate the relationship between selected clinical variables. Chi-square analysis was used to test for any significant association. Sensitivity and specificity was also calculated to find the risk level. All the statistical tests were tested with $5 \%(p \leq 0.05)$ level of significance. Follow-up DWI ASPECT score done between 48 to 72 hours after therapy was taken as the reference, as it represented the final infarct size. Correlation between baseline CT (b-CT) and baseline DWI (b-DWI) ASPECTS and 72 hours DWI were analyzed. Correlations between mRS at 90 days and ASPECT score for b-CT, b-DWI, and follow-up DWI were analyzed.

\section{Results}

This was a mixed retrospective and prospective study conducted between June 2016 and June 2018. During this period, as per the available medical records, 1844 stroke patients were seen in the hospital. For this study, we included those patients with acute ischemic stroke who underwent successful reperfusion either with endovascular thrombectomy or with intravenous (IV) thrombolysis, selected nonconsecutively from the MT database and stroke code activations, based on their angiographic reperfusion postprocedure or substantial clinical improvement following lysis, respectively.

A total of 86 patients were included in the study, which included 70 males and 16 females. Out of 86 patients, 33 patients underwent mechanical thrombectomy and 53 patients were thrombolysed. Specific time metrics were not available for the thrombolysis cohort. In the MT group, median door to first imaging was 8 minutes (interquartile range [IQR] 5-10), door to puncture was 50 minutes (IQR 4060 ), and puncture to recanalization was 60 minutes (IQR 4080). Among these, 15 underwent primary aspiration, 2 underwent stent retriever, 14 underwent combination of both (Solumbra), and 2 were given intra-arterial thrombolysis. There were 3 complications, 1 subarachnoid hemorrhage (SAH) due to wire perforation involving M2 middle cerebral artery (MCA) division and 2 nontarget embolization to anterior cerebral artery (ACA) territory. Three patients also had symptomatic intracranial hemorrhage. No patients underwent decompression among these patients. Standard criteria using both clinical and imaging details were used to decide treatment methods. Patients without intracranial large vessel occlusion were given IV thrombolysis. Among those with large vessel occlusion (33), eligible patients (22) were given IV thrombolysis before mechanical thrombectomy. Only those patients with successful reperfusion defined a priori were included in the study.

The mean age was 57.7 years in males and 61.9 years in females. b-CT ASPECTS and b-DWI ranged from 3 to 10. b-CT ASPECTS was between 3 to 5 in one patient, between 6 to 8 in 31 patients, and between 9 to 10 in 54 patients. b-DWI ASPECTS was between 3 to 5 in one patient, between 6 to 8 in 64 patients, and between 9 to 10 in 21 patients (-Table 1 ).

Out of 54 patients who had b-CT ASPECT scores between 8 to 10 , only 21 had b-DWI ASPECTS between 9 to 10 . DWI was more sensitive than CT as expected and found new infarcts in 33 patients, thereby reducing the b-DWI ASPECT score (-Figs. 2 and $\mathbf{3}$ ).

Table 1 Patient data in the different ASPECTS categories seen on baseline and follow-up imaging

\begin{tabular}{|l|l|l|l|l|}
\hline \multirow{2}{*}{$\begin{array}{l}\text { ASPECTS } \\
\text { category }\end{array}$} & Baseline & Follow-up (72 hours) \\
\cline { 2 - 5 } & $\begin{array}{l}\text { CT } \\
\text { No. of patients }\end{array}$ & $\begin{array}{l}\text { DWI } \\
\text { No. of patients }\end{array}$ & $\begin{array}{l}\text { CT } \\
\text { No. of patients }\end{array}$ & $\begin{array}{l}\text { DWI } \\
\text { No. of patients }\end{array}$ \\
\hline $3-5$ & 1 & 1 & 4 & 4 \\
\hline $6-8$ & 31 & 64 & 61 & 61 \\
\hline $9-10$ & 54 & 21 & 21 & 21 \\
\hline
\end{tabular}




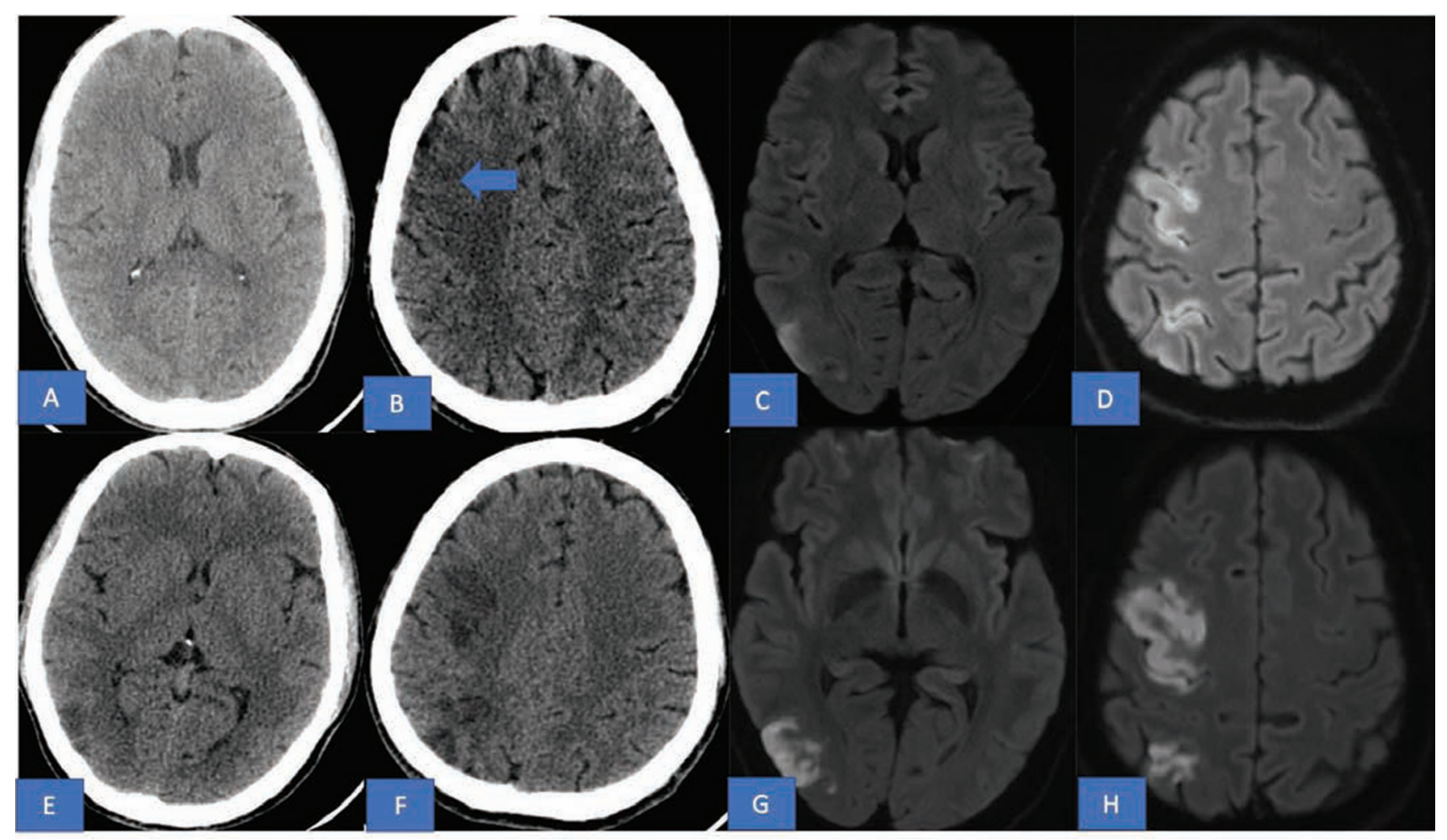

Fig. 2 Initial non contrast CT (A and B) of a middle-aged male patient at the time of clinical presentation appears unremarkable except for suspicious loss of grey-white differentiation in the right frontal region (arrow) with an ASPECT score 9. Axial diffusion MR images (C and D) however showed additional infarcts with DWI ASPECTS of 7. Subsequent 72 hours CT (E and F) shows other remote multifocal hypodensities with corresponding ASPECT score of 7. Follow up MRI with DWI $(\mathbf{G}$ and $\mathbf{H})$ confirms the final infarcts and matches the final CT ASPECTS.

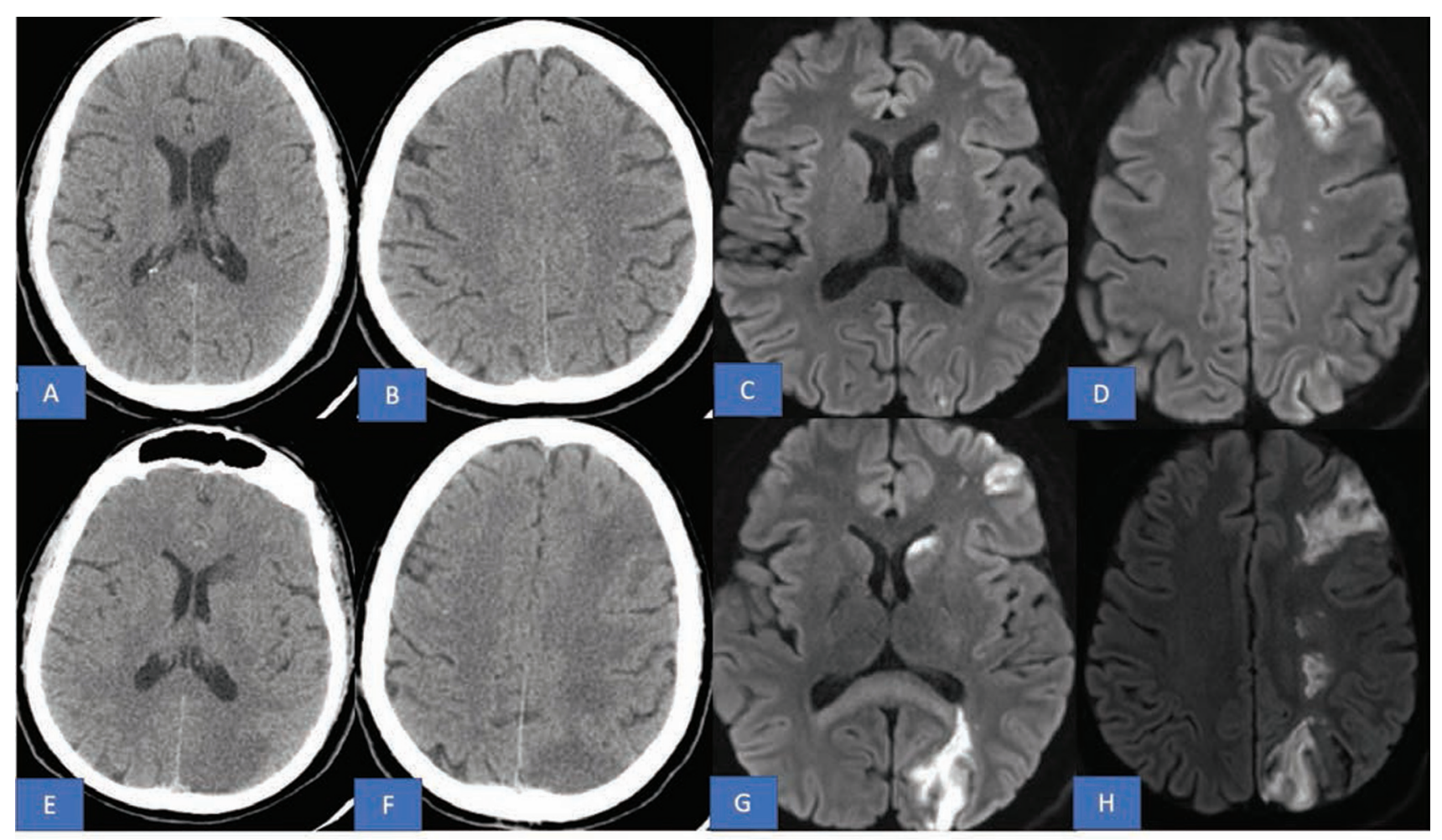

Fig. 3 Initial non contrast CT (A and B) of another patient with right sided weakness at the time of clinical presentation appears nearly unremarkable and was rated an ASPECT score of 10. However axial DWI (C and D) revealed scattered left hemispheric infarcts involving anterior and posterior watershed infarcts as well as along basal ganglia with ASPECTS of 7. Subsequent 72 hours CT (E and F) now shows established infarcts and corresponding ASPECT score of 7 . Follow up MRI ( $G$ and $\mathbf{H})$ again better demonstrates the infarcts and matches the final CT ASPECTS. 
CT and DWI ASPECTS repeated between 48 to 72 hours after therapy ranged from 3 to 10 . CT ASPECTS at 72 hours was between 3 to 5 in 4 patients, between 6 to 8 in 61 patients, and between 9 to 10 in 21 patients. DWI ASPECTS at 72 hours was between 3 to 5 in 4 patients, between 6 to 8 in 61 patients, and between 9 to 10 in 21 patients. Unlike what was seen in the pretreatment CT and DWI, there was no difference in ASPECTS between 72 hours CT and 72 hours DWI.

\section{Correlation Between b-CT and b-DWI}

Poststroke 72 hours DWI ASPECTS was taken as the reference established core, as it was deemed most suitable to represent the final infarct size.

Correlation between b-CT and b-DWI ASPECTS and 72 hours DWI were analyzed. A positive correlation of $51.2 \%$ and $84.4 \%$ was noted between b-CT ASPECTS and bDWI with 72 hours DWI ASPECTS, respectively ( $p<0.001$ ). This study shows that b-DWI ASPECTS has more positive correlation with 72 hours DWI score than b-CT ASPECTS in patients with successful reperfusion (-Fig. 4).

Correlation between mRS at 90 days and ASPECTS for bCT, b-DWI, and 72 hours DWI were analyzed. A negative correlation of $34.7 \%, 64.7 \%$ and $77.7 \%$ was noted between mRS at 90 days and ASPECTS for b-CT, b-DWI and 72 hours DWI, respectively ( $p$-value $<0.001$ ). Thus, more negative correlation was found for b-DWI and 72 hours DWI.

Sensitivity of $\mathrm{CT}$ in predicting the final infarct size was $75.3 \%$, and its specificity was $76.9 \%$. On the other hand, the sensitivity of DWI in predicting the final infarct size was $97.2 \%$, and its specificity was $92.3 \%$. The positive predictive value of CT was $94.8 \%$ and DWI was $98.6 \%$ (-Table 2).

None of the 86 patients had reversible hyperintensities in the follow-up DWI done at 72 hours.

\section{Discussion}

Imaging in stroke plays a vital role in the selection of patients for reperfusion therapy. Thus, there is a need for an imaging modality which can accurately and reliably predict the actual infarct size. Time is a crucial determinant for prognosis in
Table 2 Diagnostic accuracy of CT and MRI in acute ischemic stroke patients seen in our study

\begin{tabular}{|l|l|l|}
\hline Diagnostic performance & CT & MRI-DWI \\
\hline Sensitivity & $75.3 \%$ & $97.2 \%$ \\
\hline Specificity & $76.9 \%$ & $92.3 \%$ \\
\hline Positive predictive value & $94.8 \%$ & $98.6 \%$ \\
\hline Negative predictive value & $35.7 \%$ & $85.7 \%$ \\
\hline
\end{tabular}

stroke. The imaging modality should be chosen in such a way that the time taken for performing the scan is short. Although CT with CTA is more widely in use because of availability, MRI has already proven to be useful in selective patient populations, especially delayed presentations and in differentiation of stroke mimics. Recently, MRI has shown to identify more treatment targets in patients with minor stroke as well. It has shown that treatment pathway delays necessarily do not occur in MRI-selected patients, and it can definitely provide more information than $\mathrm{CT} .{ }^{9}$ Currently, stroke centers worldwide use either CT or MRI for selection of patients for revascularization, depending on local availability of resources.

In this study, ASPECTS were compared between the baseline and follow-up imaging done between 48 to 72 hours on CT and MRI. Our study has shown that in stroke patients presenting within 6 hours of onset of symptoms, DWI imaging has a higher positive predictive value in predicting the final infarct size than noncontrast $\mathrm{CT}$, as shown in the representative images (-Figs. 2 and 3 ). Sensitivity and specificity of DWI in picking up infarct is higher than noncontrast CT. Sensitivity and specificity of DWI-MRI was $97.2 \%$ and $92.3 \%$, respectively.

Nezu et al showed that DWI ASPECTS was one point lower than CT ASPECTS, which implies that DWI ASPECTS is more sensitive than $\mathrm{CT}$ in predicting the infarct size. ${ }^{10} \mathrm{~A}$ study done by Barber et al showed that DWI is more sensitive in identifying areas of infarct than noncontrast CT. This study did not use ASPECTS for measuring infarct size. ${ }^{11}$ Ryan et al used ASPECTS for estimating the infarct size, and analyzed the interrater agreement for DWI ASPECTS and CT ASPECTS.

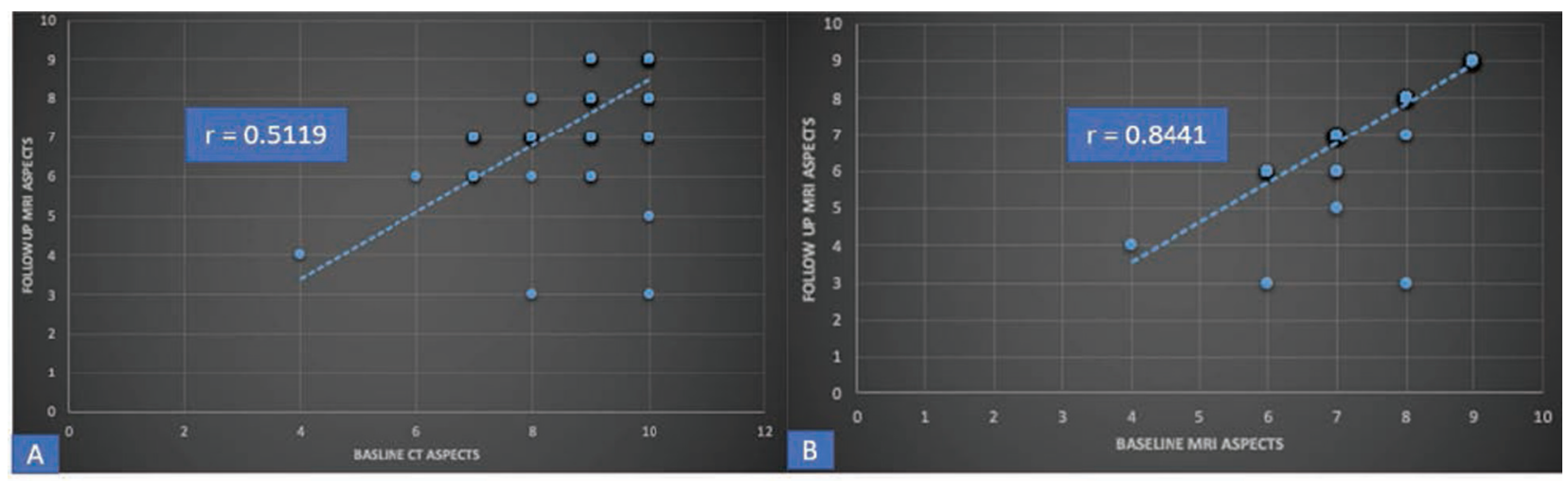

Fig. 4 Scatter plot diagrams showing correlation between final infarct burden as seen on follow-up diffusion-weighted imaging (DWI) versus baseline CT (A) and baseline DWI (B) Alberta stroke programme early CT score (ASPECTS), respectively. 
Table 3 Comparison of previous studies

\begin{tabular}{|c|c|c|c|}
\hline Study & CT & DWI & Functional outcome \\
\hline Barber et al ${ }^{11}$ & $\mathrm{CT}=\mathrm{DWI}$ & $\mathrm{CT}=\mathrm{DWI}$ & \\
\hline Nezu et al ${ }^{10}$ & $\begin{array}{l}\text { Less sensitive for ischemic change in } \\
\text { caudate and internal capsule }\end{array}$ & $\begin{array}{l}\text { More sensitive } \\
(1 \text { point less than } \mathrm{CT} \text { ) }\end{array}$ & $C T=D W I$ \\
\hline Ryan et $\mathrm{al}^{12}$ & Less sensitive & More sensitive & $\mathrm{DWI}>\mathrm{CT}$ \\
\hline Mitomi et al ${ }^{13}$ & $\begin{array}{l}\text { Low sensitivity for internal } \\
\text { capsule and caudate nucleus }\end{array}$ & More sensitive & Not done in the study \\
\hline Hiroyuki et al $^{14}$ & & $\begin{array}{l}\text { May underestimate acute } \\
\text { ischemic lesions }\end{array}$ & - \\
\hline
\end{tabular}

Abbreviation: DWI, diffusion-weighted imaging.

They found that interrater agreement was superior for DWI ASPECTS as compared to CT ASPECTS. ${ }^{12}$ Mitomi et al compared the sensitivity and specificity of ASPECTS for CT and DWI and found that CT showed low sensitivity and specificity in identifying areas of infarct. This study was done in patients presenting within 3 hours of onset of stroke. ${ }^{13}$

A study done by Barber et al showed that a very small difference exists between CT and MRI in establishing acute infarct. As CT is faster and easily accessible, this study concluded that $\mathrm{CT}$ is a better imaging modality in acute stroke. ${ }^{14}$ The disadvantage of this study was prolonged time taken between the two imaging modalities. This was overcome in the study done by Nezu et al where the time interval between CT and MRI was short. In our study, the average time interval between the two modalities was 20 minutes, which is comparable to the study done by Nezu et al (-Table 3 ).

Our study has shown that there is a significant negative correlation between CT and MRI ASPECTS (at baseline and follow-up between 48 to 72 hours after therapy) and functional outcome at 90 days. Correlation was higher for DWI than for CT. Higher the score in DWI ASPECTS, lower the mRS. This implies a good functional outcome for higher DWI ASPECTS. Thus, DWI ASPECTS can be used as an indicator of functional outcome. Our results are similar to the study done by Mc Taggart et al in which it was found that DWI ASPECTS correlated well with the functional outcome at 90 days than noncontrast CT. Nezu et al found that the area under curve for DWI ASPECTS was on the higher side than for CT ASPECTS and can be used as a better predictor of functional outcome than CT ASPECTS.

In our study, none of the patients showed reversible hyperintensities in follow-up DWI. Although few previous studies $^{15}$ have shown that some of the DWI changes can reverse, dichotomization of DWI will give us a fair estimate of the final infarct size, and minor changes are unlikely to change treatment decisions. Baseline MRI can fairly, even if not always, represent true regions of the brain, which are irreversibly dead, and not the ischemic tissue. A previous study done by Chemmanam et al also showed no significant reversal of diffusion hyperintensities; even if present, they are too small to alter decision-making. Other previous studies on reversal of diffusion showed that diffusion reversal was rarely found in a few patients who had smaller baseline infarct volumes and was more common in embolic stroke patients There was no diffusion reversal in patients who had large volumes of infarct and mRS $>2$ at 90 days. Both these studies were done in $3 \mathrm{~T}$ scanner. Reversibility of diffusion hyperintensities is still controversial. ${ }^{16,17}$ Further studies are needed for validation. The correlation between the strength of the magnetic field and reversibility of diffusion has to be studied. However, the available literature shows that the impact of these reversible hyperintensities in deciding the treatment is very small. Thus, decision-making for appropriate treatment can be based on the DWI ASPECTS, without any risk of overestimating the infarct size as the benefit-risk ratio is high. Penumbra is indicated by the mismatch between clinical presentation and imaging correlates of definitive core. Both CT and DWI ASPECTS aim to demonstrate the core, and their difference cannot be interpreted as the penumbra. However, their mismatch with clinical presentation (NIHSS) can be surrogate marker of penumbra. Definite advantages of MRI over CT in acute stroke, apart from being more sensitive for infarct burden, include ability to differentiate stroke mimics using patterns, noncontrast evaluation of the craniocervical vessels as well as tissue perfusion (ASL), and tissue-based timing using DWI-FLAIR mismatch in patients with unknown time of onset and lack of radiation.

\section{Limitations}

Clinical outcome in stroke depends on the region of the brain affected. Our study did not analyze the differences in clinical outcome for eloquent and noneloquent areas separately. Individual time metrics for the thrombolysis patients were not available. Infarct size or volume mapping was not done, and ASPECTS were correlated to the infarct burden. No vascular imaging modality was used in patients who underwent successful thrombolysis to confirm recanalization. Lack of inclusion of all consecutive ischemic stroke patients was another limitation of this study. This study also did not look into the impact of b-DWI on treatment decisions. Purpose of the study was to look at the diagnostic accuracy of the different imaging modalities in predicting final infarct burden, and being nonconsecutive samples, it is difficult to draw conclusions on clinical outcomes. Within the individual groups, association between DWI ASPECTS at 72 hours and 
90 day mRS was highly significant ( $p<0.00001)$. Future studies enrolling consecutive patients are needed to validate these results in larger prospective cohort as well as look into various other details mentioned above.

\section{Knowledge Gaps and Future Directions}

Although MRI is known to be more sensitive to detect infarcts than CT in stroke patients, real-world data comparing CT and MRI, especially in patients undergoing acute recanalization therapies, are scarce due to nonavailability of MRI in the emergency settings as well as widespread utilization of CT and CTA for decision-making following various endovascular trials. Only one endovascular trial directly compared MRI versus CT in acute stroke prior to mechanical thrombectomy. ${ }^{6}$ They found that MRI scan duration was slightly longer than CT; however, it can be accomplished without delaying treatment or impacting functional outcomes. Future pragmatic randomized controlled trials enrolling consecutive patients are needed to compare both the modalities in acute stroke prior to treatment, and till such time, these studies add to the existing knowledge gap in this domain. Even collateral assessment, which currently depends on multiphasic CTA, can have surrogate markers of on MRI such as FLAIR (hyperintense vessel sign [HVS]), ASL (arterial transit artefacts/signals [ATA]) as well as mismatch with core (DWIASPECTS), apart from looking at postcontrast dynamic MR angiography in future. Thus, all hope is still not lost for MRI in acute stroke,and we do have a chance to correct the wrongs. ${ }^{18}$

\section{Conclusions}

DWI is more sensitive and specific than noncontrast CT in predicting final infarct volume and can be used as a predictor of functional outcome. However, CT has a major role to rule out acute bleed, as it is fast and reliable. Combination of CT and DWI is the best and fast imaging modality available, to date, for predicting the final infarct size and assessing the penumbra. MRI predicts the final outcome better and should be used for better selection of patients for recanalization. Ideally, if local logistics permit, a combination of CT and DWI can help in best predicting the final infarct size, since the sensitivity of CT is less and the latter can sometimes show reversal, although not seen in this study.

Conflict of Interest

None declared.

\section{References}

1 Kamalakannan S, Gudlavalleti ASV, Gudlavalleti VSM, Goenka S, Kuper H. Incidence \& prevalence of stroke in India: a systematic review. Indian J Med Res 2017;146(02):175-185

2 Powers WJ, Rabinstein AA, Ackerson T, et al. Guidelines for the Early Management of Patients With Acute Ischemic Stroke: 2019
Update to the 2018 Guidelines for the Early Management of Acute Ischemic Stroke: A Guideline for Healthcare Professionals From the American Heart Association/American Stroke Association. Stroke 2019;50(12):e344-e418

3 Nogueira RG, Jadhav AP, Haussen DC, et al. DAWN Trial Investigators. Thrombectomy 6 to 24 hours after stroke with a mismatch between deficit and infarct. N Engl J Med 2018;378 (01):11-21

4 Albers GW, Marks MP, Kemp S, et al. DEFUSE 3 Investigators. Thrombectomy for stroke at 6 to 16 hours with selection by perfusion imaging. N Engl J Med 2018;378(08):708-718

5 Kang D-W, Sohn S-I, Hong K-S, et al. Reperfusion therapy in unclear-onset stroke based on MRI evaluation (RESTORE): a prospective multicenter study. Stroke 2012;43(12):3278-3283

6 Provost C, Soudant M, Legrand L, et al. Magnetic resonance imaging or computed tomography before treatment in acute ischemic stroke. Stroke 2019;50(03):659-664

7 Fugate JE, Klunder AM, Kallmes DF. What is meant by “TICI”? AJNR Am J Neuroradiol 2013;34(09):1792-1797

8 Rudilosso S, Urra X, Amaro S, et al. Timing and relevance of clinical improvement after mechanical thrombectomy in patients with acute ischemic stroke. Stroke 2019;50(06):1467-1472

9 Hsia AW, Luby ML, Leigh R, et al. Prevalence of imaging targets in patients with minor stroke selected for IV tPA treatment using mri: the treatment of minor stroke with MRI evaluation study (TIMES. Neurology 2021;96(09):e1301-e1311

10 Nezu T, Koga M, Nakagawara J, et al. Early ischemic change on CT versus diffusion-weighted imaging for patients with stroke receiving intravenous recombinant tissue-type plasminogen activator therapy: stroke acute management with urgent risk-factor assessment and improvement (SAMURAI) rt-PA registry. Stroke 2011;42(08):2196-2200

11 Barber PA, Hill MD, Eliasziw M, et al. ASPECTS Study Group. Imaging of the brain in acute ischaemic stroke: comparison of computed tomography and magnetic resonance diffusionweighted imaging. J Neurol Neurosurg Psychiatry 2005;76(11): 1528-1533

12 McTaggart RA, Jovin TG, Lansberg MG, et al. DEFUSE 2 Investigators. Alberta stroke program early computed tomographic scoring performance in a series of patients undergoing computed tomography and MRI: reader agreement, modality agreement, and outcome prediction. Stroke 2015;46(02): 407-412

13 Mitomi M, Kimura K, Aoki J, Iguchi Y. Comparison of CT and DWI findings in ischemic stroke patients within 3 hours of onset. J Stroke Cerebrovasc Dis 2014;23(01):37-42

14 Kawano H, Hirano T, Nakajima M, Inatomi Y, Yonehara T. Diffusion-weighted magnetic resonance imaging may underestimate acute ischemic lesions: cautions on neglecting a computed tomography-diffusion-weighted imaging discrepancy. Stroke 2013; 44(04):1056-1061

15 Chemmanam T, Campbell BCV, Christensen S, Nagakane Y, Desmond PM, Bladin CF, et al. Ischemic diffusion lesion reversal is uncommon and rarely alters perfusion-diffusion mismatch. Neurology 2010;75(12):1040-1047

16 Nagaraja N, Forder JR, Warach S, Merino JG. Reversible diffusionweighted imaging lesions in acute ischemic stroke: A systematic review. Neurology 20203194(13):571-587

17 Asdaghi N, Campbell BCV, Butcher KS, et al. DWI Reversal Is Associated with Small Infarct Volume in Patients with TIA and Minor Stroke. Am J Neuroradiol 2014;35(04):660-666

18 Lövblad KO, Pereira VM. MRI of Acute Stroke: What Went Wrong? AJNR Am J Neuroradiol 2015;36(11): 1996-1997 